OPEN ACCESS

Edited by:

Yaohua Liu,

Shanghai First People's Hospital,

China

Reviewed by:

Kristin Schroeder,

Duke Cancer Institute, United States

Gerardo Caruso,

University Hospital of Policlinico G.

Martino, Italy

*Correspondence:

Gang Zhao

gzhao@jlu.edu.cn

Yubo Wang

wangyubo@jlu.edu.cn

${ }^{t}$ These authors have contributed equally to this work and share first authorship

Specialty section: This article was submitted to Neuro-Oncology and

Neurosurgical Oncology, a section of the journal

Frontiers in Oncology

Received: 07 April 2021

Accepted: 26 May 2021

Published: 11 June 2021

Citation:

Liu H, Qin X, Zhao L, Zhao G and Wang $Y$ (2021) Epidemiology and Survival of Patients With Brainstem Gliomas: A Population-Based Study Using the SEER Database.

Front. Oncol. 11:692097. doi: 10.3389/fonc.2021.692097

\section{Epidemiology and Survival of Patients With Brainstem Gliomas: A Population-Based Study Using the SEER Database}

\author{
Huanbing Liu ${ }^{1 \dagger}$, Xiaowei Qin ${ }^{1 \dagger}$, Liyan Zhao ${ }^{2}$, Gang Zhao ${ }^{1 *}$ and Yubo Wang ${ }^{1 *}$ \\ 1 Department of Neurosurgery, First Hospital of Jilin University, Changchun, China, ${ }^{2}$ Department of Clinical Laboratory, \\ Second Hospital of Jilin University, Changchun, China
}

Background: Brainstem glioma is a primary glial tumor that arises from the midbrain, pons, and medulla. The objective of this study was to determine the population-based epidemiology, incidence, and outcomes of brainstem gliomas.

Methods: The data pertaining to patients with brainstem gliomas diagnosed between 2004 and 2016 were extracted from the SEER database. Descriptive analyses were conducted to evaluate the distribution and tumor-related characteristics of patients with brainstem gliomas. The possible prognostic indicators were analyzed by Kaplan-Meier curves and a Cox proportional hazards model.

Results: The age-adjusted incidence rate was 0.311 cases per 100,000 person-years between 2004 and 2016. A total of 3387 cases of brainstem gliomas were included in our study. Most of the patients were white and diagnosed at 5-9 years of age. The most common diagnosis confirmed by histological review was ependymoma/anaplastic ependymoma. The median survival time was 24 months. Patients with tumors less than $3 \mathrm{~cm}$ in size had a better prognosis. Surgery was effective at improving overall survival. There was no evidence that radiotherapy and chemotherapy improved overall survival.

Conclusion: Brainstem gliomas can be diagnosed at any age. Ependymoma/anaplastic ependymoma is the most common pathological diagnosis. The prognosis is poor, and timely diagnosis and surgery are effective at improving the prognosis. We suggest that more attention should be given to the treatment of patients with brainstem gliomas.

Keywords: brainstem glioma, epidemiology, survival, SEER Program, CNS disease

\section{INTRODUCTION}

Gliomas are primary brain tumors that are thought to arise from neuroglial stem or progenitor cells (1). Gliomas are the most common malignant primary brain tumor, and $4.3 \%$ of gliomas are localized at the brainstem (2). Brainstem glioma is a primary glial tumor that arises from the midbrain, pons, and medulla. In most instances, the term refers to a highly aggressive tumor of the pons (3). Diffuse intrinsic pontine glioma has been reported to account for $\sim 75 \%$ of brain stem 
tumors in children (2). Because of poor survival, brainstem glioma has been a main research focus for decades (4).

The Surveillance, Epidemiology, and End Results (SEER) program of the National Cancer Institute represents approximately 35\% of the US population (based on the 2000 census) (5). Our goal is to use SEER data to analyze the epidemiology and survival of patients with brainstem gliomas in the United States.

\section{METHODS}

\section{Data Extraction and Incidence Rates}

The data from SEER are available to the public for research purposes. Therefore, ethics committee approval and informed consent were not necessary to perform the analyses. Patients with a diagnosis of primary brainstem gliomas were included. The term glioma was defined by setting the variable "Histology recode - broad groupings" as "9380-9489: gliomas". Brainstem gliomas were defined by setting the variable "Primary Site labeled" as "C71.7-Brainstem". The research period was set from 2004 to 2016. Age-adjusted incidence rates (directly standardized to the 2000 US standard population) between 2004 and 2016 were retrieved from the SEER 18 database (November 2019 submission) (6). The detailed patient data were obtained from SEER 18 Regs Custom Data (November 2018 submission) (7). All the data were obtained by using the SEER ${ }^{\star}$ Stat 8.3.8 program.

\section{Variables and Population Analysis}

The demographic and clinical features included age at diagnosis (019 years, $\geq 20$ years), sex (Male, Female), race (White, Black, Asian or Pacific Islander, American Indian/Alaska Native, Unknown), Purchased/Referred Care Delivery Area (PRCDA) Region (Alaska, eastern region, north plains, Pacific coast, southwestern region), tumor size ( $\leq 3 \mathrm{~cm},>3 \mathrm{~cm}$ and Unknown), diagnostic confirmation (positive microscopic confirm, others), behavior code (benign, borderline malignancy, malignant) according to the International Classification of Diseases for Oncology 3 (ICD-O-3), surgery (Yes, None/Unknown), radiation therapy (Yes, None/Unknown), chemotherapy (Yes, None/Unknown), survival months and vital status. The pathology types (according to the code of "Histology recode - Brain groupings") of the patients diagnosed with positive microscopic confirmation were analyzed. Descriptive analyses were conducted to evaluate the distribution and tumor-related characteristics of patients with brainstem gliomas. Bar graphs and pie charts were also used to further describe the distribution of patients.

\section{Survival Analysis}

The Kaplan-Meier method was used to estimate overall survival (OS) at 1, 3, 5, and 10 years. Survival time was defined as the time from diagnosis to death from any cause. We also used this method to estimate the OS in different groups. The differences between the curves were analyzed by the log-rank test. Univariate and multivariate Cox proportional hazard models were performed to estimate the hazard ratios (HRs) and 95\% confidence intervals (CIs) to analyze the independent prognostic factors associated with OS in patients with brainstem gliomas, and statistical significance was defined as $\mathrm{p}<0.05$. All the data were analyzed by IBM SPSS Statistics 25 software (IBM Corporation, Armonk, New York, USA).

\section{RESULTS}

\section{Population Analysis}

The age-adjusted incidence rate was 0.311 cases per 100,000 person-years between 2004 and 2016. A total of 3387 cases of brainstem gliomas were indexed between 2004 and 2016. The demographic and clinical characteristics of the patients are shown in Table 1. There were 1535 female patients (45.3\%) and 1852 male patients (54.7\%). The median age was 18 years (range 0 to 103 years). The majority of patients were diagnosed when they were between 5 and 9 years old, and the distribution of patient age at diagnosis is shown in a histogram (Figure 1). Children and adolescents ( $0-19$ years old) accounted for $34.3 \%$ of all patients. White patients accounted for $80.2 \%$ of all patients (Figure 2). Most of the patients were from the Pacific coast $(n=1740,51.4 \%)$ and eastern region $(n=1187,35.0 \%)$. According to ICD-0-3, most of the tumors were malignant $(n=3040,89.8 \%)$. Among this cohort, 2023 cases were diagnosed with positive microscopic confirmation. We analyzed pathology type among these patients, and the results are shown in a pie chart (Figure 3). We found that ependymoma/anaplastic ependymoma $(n=438$, $21.7 \%)$ and pilocytic astrocytoma $(n=377,18.6 \%)$ were the most common pathology types. A total of $37.2 \%$ of the tumors were less than $3 \mathrm{~cm}$ in size, and $33.5 \%$ of the tumors were larger than $3 \mathrm{~cm}$. Radiation was the first choice of therapy for patients with brainstem gliomas. Surgery was performed in 1479 (43.7\%) cases, radiation therapy was performed in 1746 (51.6\%) cases, and chemotherapy was performed in 1166 (34.4\%) cases. The median survival time was 24 months (range 0 to 155 months). At the time of data collection, 1931 (57.0\%) patients were alive, and $1456(43.0 \%)$ were deceased.

\section{Survival Analysis}

The OS rates at $1,3,5$ and 10 years after diagnosis were $70.8 \%$, $56.3 \%, 53.3 \%$ and $48.8 \%$, respectively. A Kaplan-Meier curve was created to show the OS for the full cohort (Figure 4A). The Kaplan-Meier log-rank test indicated that the variables age at diagnosis (Figure 4B), race (Figure 4C), tumor size (Figure 4D), behavior (Figure 4E), surgery (Figure 4F), radiation (Figure 4G) and chemotherapy (Figure $\mathbf{4 H}$ ) were possibly related to OS. The results of multivariate Cox proportional hazard regression analysis showed that race, age and sex were not independent prognostic factors. Patients with tumors less than $3 \mathrm{~cm}$ in size or benign or borderline tumors had a better prognosis. The results also showed that surgery was effective for improving prognosis, but radiation and chemotherapy did not help patients obtain a better prognosis. The results generated by the log-rank test and univariate and multivariate Cox proportional hazard models are listed in Table 2. 
TABLE 1 | Demographic and clinical characteristics of patients with brainstem gliomas.

\begin{tabular}{|c|c|c|c|}
\hline Variables & Number & & $\%$ \\
\hline \multicolumn{4}{|l|}{ Sex } \\
\hline Female & 1535 & & 45.3 \\
\hline Male & 1852 & & 54.7 \\
\hline \multicolumn{4}{|l|}{ Age at diagnosis (years) } \\
\hline Mean \pm SD & \multicolumn{3}{|c|}{$26.86 \pm 23.244$} \\
\hline Median & \multicolumn{3}{|c|}{18.00} \\
\hline Range & \multicolumn{3}{|c|}{$0-103$} \\
\hline $0-19$ & 1161 & & 34.3 \\
\hline$\geq 20$ & 2226 & & 65.7 \\
\hline \multicolumn{4}{|l|}{ Race } \\
\hline White & 2715 & & 80.2 \\
\hline Others & 672 & & 19.8 \\
\hline \multicolumn{4}{|l|}{ PRCDA Region } \\
\hline Alaska & 6 & & 0.2 \\
\hline East & 1187 & & 35.0 \\
\hline North plains & 258 & & 7.6 \\
\hline Pacific coast & 1740 & & 51.4 \\
\hline Southwest & 196 & & 5.8 \\
\hline \multicolumn{4}{|l|}{ Tumor Size } \\
\hline$\leq 3 \mathrm{~cm}$ & 1259 & & 37.2 \\
\hline$>3 \mathrm{~cm}$ & 1134 & & 33.5 \\
\hline Unknown & 994 & & 29.3 \\
\hline \multicolumn{4}{|l|}{ Diagnostic confirmation } \\
\hline Positive microscopic confirm & 2023 & & 59.7 \\
\hline Others & 1364 & & 40.3 \\
\hline \multicolumn{4}{|l|}{ Behavior code } \\
\hline Benign & 113 & & 3.3 \\
\hline Borderline malignancy & 234 & & 6.9 \\
\hline Malignant & 3040 & & 89.8 \\
\hline \multicolumn{4}{|l|}{ Surgery } \\
\hline Yes & 1479 & & 43.7 \\
\hline None/Unknown & 1908 & & 56.3 \\
\hline \multicolumn{4}{|l|}{ Radiation } \\
\hline Yes & 1746 & & 51.6 \\
\hline None/Unknown & 1641 & & 48.4 \\
\hline \multicolumn{4}{|l|}{ Chemotherapy } \\
\hline Yes & 1166 & & 34.4 \\
\hline None/Unknown & 2221 & & 65.6 \\
\hline \multicolumn{4}{|l|}{ Survival months } \\
\hline Mean $\pm S D$ & & $44.22 \pm 44.404$ & \\
\hline Median & & 24.00 & \\
\hline Range & & $0-155$ & \\
\hline \multicolumn{4}{|l|}{ Vital status } \\
\hline Alive & 1931 & & 57.0 \\
\hline Dead & 1456 & & 43.0 \\
\hline
\end{tabular}

\section{DISCUSSION}

Brainstem glioma is a primary glial tumor that arises within the brainstem and is believed to be a heterogeneous group of gliomas. Some authors divided brainstem gliomas into 2 categories. Twenty percent are considered to be focal lowgrade lesions with good prognosis, and the remaining $80 \%$ of tumors arise in and occupy the majority of the pons and are diffuse in nature and associated with poor prognosis $(8-10)$. We could not define whether the tumor originated from the midbrain, medulla, or pons, and we also could not define whether the tumor was focal or diffuse based on the data available in the SEER database. Some authors have conducted cohort studies with data on high-grade glioma $(11,12)$ or low- grade glioma (13) based on the SEER database. Brainstem glioma is a relative rare lesion, and previous reports have included limited numbers of cases. We believe that this limitation might prevent us from obtaining a better understanding of the general characteristics of the patients diagnosed with brainstem glioma. The SEER program represents approximately $35 \%$ of the US population, and the data were collected from most parts of the United States (5). To better understand brainstem glioma, we conducted this large-scale cohort study including 3387 cases. To the best of our knowledge, this is the largest brainstem glioma cohort to date. We believe our report can explain the epidemiology and survival of patients with brainstem gliomas in the United States to some extent.

First, we wanted to conduct an analysis of cancer-specific survival. However, because the brainstem glioma was not the first malignant tumor in some patients, cancer-specific survival was not applicable for approximately $10 \%$ of the cohort. Progressionfree survival cannot be adopted because we cannot obtain data about progression from the SEER database. To include all of the data and make the analysis accurate, we chose to analyze OS. The World Health Organization (WHO) grade classification is widely used by neuro-oncology doctors, but half of the tumors were not clearly classified according to WHO grade. Therefore, we chose the behavior code according to ICD-O-3; in our cohort, more than $80 \%$ of the tumors were malignant, which is in agreement with a previous study (3).

According to the report from The Central Brain Tumor Registry of the United States (14), in children and adolescents, brainstem tumors account for $10.8 \%$ of all primary central nervous system tumors. Brainstem gliomas were also reported to account for up to $20 \%$ or more of primary brain tumors (15). In our cohort, the highest incidence was found in individuals between 5 and 9 years old, and the median age at diagnosis was 18 years old. However, we found that brainstem gliomas could be diagnosed at every age. Children and adolescents ( 0 - 19 years old) accounted for $34.3 \%$ of all patients, and more than half of the patients were adults. The predominance of white patients was also very significant in our study, which is consistent with the findings of other cohort studies of gliomas based on the SEER program $(13,16)$. Males and females are almost equally affected. We have also analyzed the pathological classification of the tumors in these patients, with the intention of providing information that could be useful when biopsy or surgery cannot be performed but a treatment needs to be selected. According to a previous report, diffuse intrinsic tumors account for approximately $80 \%$ of all brainstem gliomas. These tumors are generally high-grade anaplastic astrocytoma (WHO grade 3 ), glioblastoma multiforme (WHO grade 4 ), or occasionally well-differentiated diffuse astrocytoma (WHO grade 2) (17). However, in our cohort, we found that the most common pathology type was ependymoma/anaplastic ependymoma and pilocytic astrocytoma. Glioblastoma accounted for only $9 \%$ of all cases with positive histology. Because the anatomical location of the tumor precludes surgery, radiation is the standard therapy for patients with diffuse intrinsic tumors (17), and tumor resection or biopsy is 

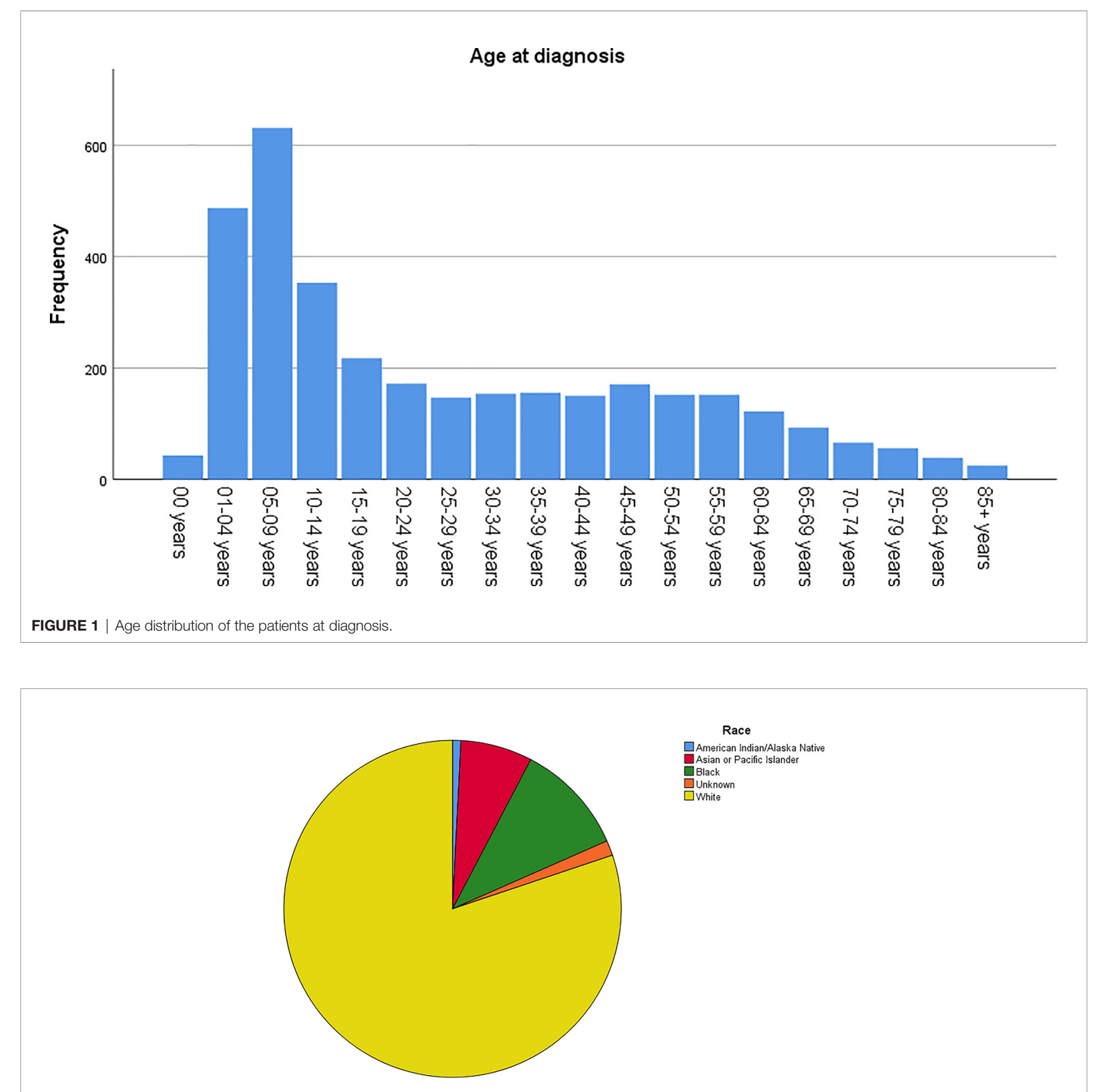

FIGURE 2 | Racial distribution of the patients.

not recommended for some patients. In our cohort, $59.7 \%$ of the cases were diagnosed with a positive microscopic method. There could be some bias in our research of the pathological characteristics because the data were not fully included. We believe further investigation should be conducted to clarify the findings, and further research about molecular pathology is also needed.

In our study, we found that race, age and sex were not independent prognostic factors. However, patients with tumors less than $3 \mathrm{~cm}$ in size had a better prognosis than patients with tumors larger than $3 \mathrm{~cm}$. We believe that timely diagnosis and treatment are essential for patients. Focal radiation therapy is the current standard of care for children with diffuse intrinsic pontine glioma (18). We also found that radiation therapy was chosen more frequently than surgery and chemotherapy. However, the results of the survival analysis showed that radiation and chemotherapy did not improve overall survival. The systematic review conducted by Xu et al. (19) also could not make definitive conclusions regarding whether radiotherapy can help patients obtain better survival. We believe that further high- 


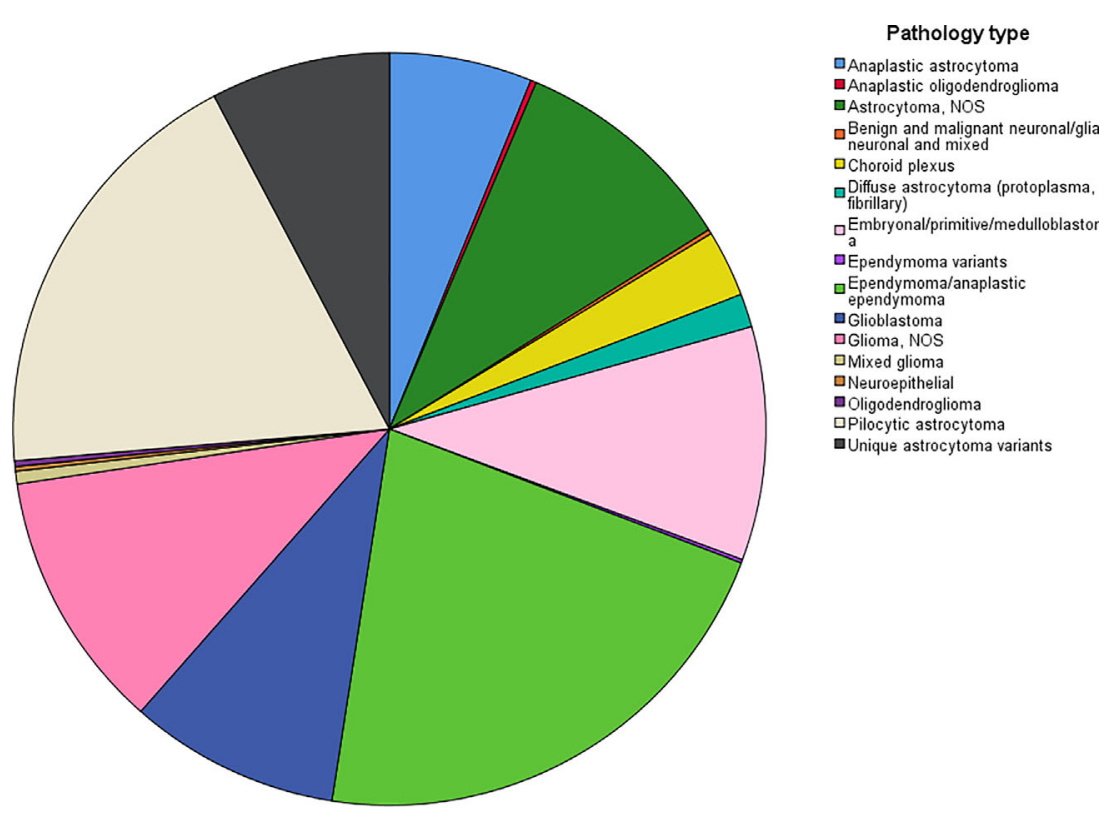

FIGURE 3 | The distribution of the patients with different pathological types.

A

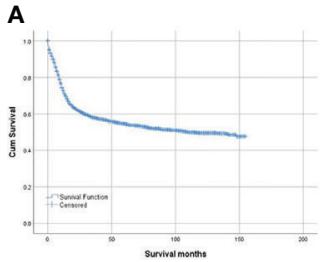

E

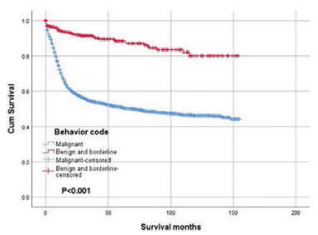

B

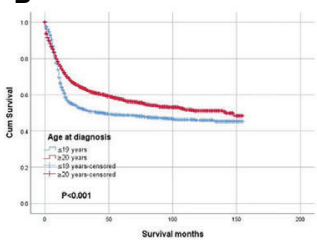

$\mathbf{F}$

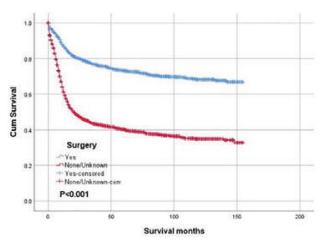

C

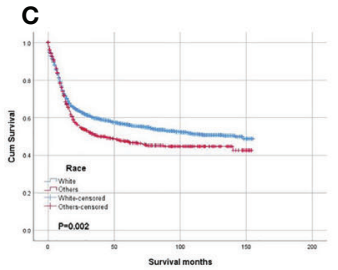

G

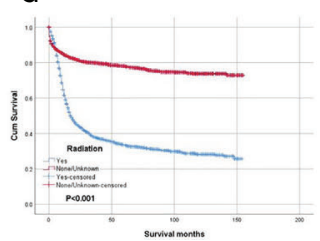

D

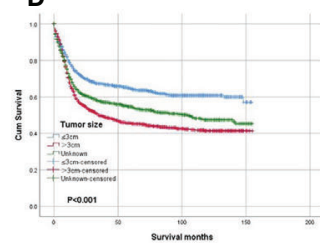

H

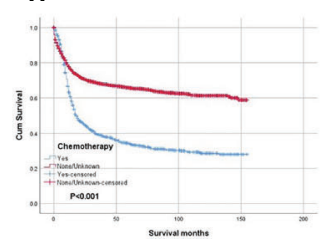

FIGURE 4 | Kaplan-Meier survival analysis: (A) The overall survival for the whole cohort. The survival analysis of patients classified based on (B) age at diagnosis, (C) race, (D) tumor size, (E) behavior, (F) surgery, (G) radiation and $(\mathbf{H})$ chemotherapy.

quality studies are needed to establish the role of radiotherapy in the management of brainstem gliomas. Brainstem glioma is believed to be composed of a heterogeneous group of gliomas, and individualized treatment is needed based on pathology. Our results also showed that surgery can improve prognosis. The extent of surgery in some cases was difficult to clarify based on the code provided. In particular, biopsy and ventricular peritoneal shunts are widely used for patients with brainstem gliomas. We did not analyze the impact of surgery extent on survival. Based on the results, the prognosis of brainstem glioma is poor, and the median survival time is 24 months. Different attempts have been made to treat patients with brainstem gliomas $(4,18,20)$, and we hope that more effective treatments can be discovered in the future.

There are several limitations of our analysis that must be considered. The availability of some important information was limited, such as information on the tumor location (midbrain, pons, and medulla), focality of diffuse glioma, molecular pathology, more specific treatment, tumor progression and so on. The specific location and molecular features are very important factors affecting progression, and molecular pathological investigations have been widely implemented in clinical practice. We hope that we can obtain more detailed information from the SEER database in the future. Although it would necessitate the inclusion of a 
TABLE 2 | Results of the log-rank test and univariate and multivariate Cox regression analysis.

\begin{tabular}{|c|c|c|c|c|c|}
\hline \multirow[t]{2}{*}{ Variable } & \multirow{2}{*}{$\begin{array}{l}\text { Log-Rank Test } \\
\text { P value }\end{array}$} & \multicolumn{2}{|c|}{ Univariate Analysis } & \multicolumn{2}{|c|}{ Multivariate Analysis } \\
\hline & & HR $(95 \% \mathrm{Cl})$ & $P$ value & HR $(95 \% \mathrm{Cl})$ & $P$ value \\
\hline Sex & 0.270 & & & & \\
\hline Female & & Reference & & Reference & \\
\hline Male & & $0.944(0.851-1.047)$ & 0.275 & 0.998 (0.899-1.108) & 0.973 \\
\hline Age at diagnosis (years) & $<0.001$ & & & & \\
\hline$\leq 19$ & & Reference & & Reference & \\
\hline$\geq 20$ & & $0.802(0.721-.892)$ & $<0.001$ & 0.908 (0.812-1.016) & 0.092 \\
\hline Race & 0.002 & & & & \\
\hline White & & Reference & & Reference & \\
\hline Others/Unknown & & $1.210(1.069-1.370)$ & 0.003 & 1.003 (0.885-1.137) & 0.959 \\
\hline Tumor Size & $<0.001$ & & & & \\
\hline$\leq 3 \mathrm{~cm}$ & & Reference & & Reference & \\
\hline$>3 \mathrm{~cm}$ & & $1.662(1.462-1.889)$ & $<0.001$ & $1.240(1.079-1.424)$ & 0.002 \\
\hline Unknown & & 1.408 (1.227-1.616) & $<0.001$ & 1.159 (1.008-1.332) & 0.038 \\
\hline Behavior & $<0.001$ & & & & \\
\hline Malignant & & Reference & & Reference & \\
\hline Benigan and Borderline & & $0.212(0.155-0.290)$ & $<0.001$ & 0.507 (0.366-0.703) & $<0.001$ \\
\hline Surgery & $<0.001$ & & & & \\
\hline None/Unknown & & Reference & & Reference & \\
\hline Yes & & 0.337 (0.299-0.380) & $<0.001$ & $0.337(0.298-0.381)$ & $<0.001$ \\
\hline Radiation & $<0.001$ & & & & \\
\hline None/Unknown & & Reference & & Reference & \\
\hline Yes & & $3.651(3.235-4.121)$ & $<0.001$ & $2.832(2.465-3.254)$ & $<0.001$ \\
\hline Chemotherapy & $<0.001$ & & & & \\
\hline None/Unknown & & Reference & & Reference & \\
\hline Yes & & $2.188(1.972-2.428)$ & 0.001 & $1.234(1.100-1.384)$ & $<0.001$ \\
\hline
\end{tabular}

substantial amount of information, it would assist in the realization of a better understanding of primary CNS tumors.

\section{CONCLUSION}

Brainstem gliomas can be diagnosed at every age. Ependymoma/ anaplastic ependymoma is the most common pathological diagnosis. The predominance of white patients was significant. The prognosis was poor, and the median survival time was 24 months. Timely diagnosis and surgery are effective in improving the prognosis, and individualized treatment is essential for patients. We suggest that more attention should be paid to the treatment of patients with brainstem gliomas.

\section{REFERENCES}

1. Weller M, Wick W, Aldape K, Brada M, Berger M, Pfister SM, et al. Glioma. Nat Rev Dis Primers (2015) 1:15017. doi: 10.1038/nrdp.2015.17

2. Ostrom QT, Patil N, Cioffi G, Waite K, Kruchko C, Barnholtz-Sloan JS. Cbtrus Statistical Report: Primary Brain and Other Central Nervous System Tumors Diagnosed in the United States in 2013-2017. Neuro-Oncology (2020) 22:iv1-96. doi: 10.1093/neuonc/noaa200

3. Grimm SA, Chamberlain MC. Brainstem Glioma: A Review. Curr Neurol Neurosci Rep (2013) 13:346. doi: 10.1007/s11910-013-0346-3

4. Chen LH, Pan C, Diplas BH, Xu C, Hansen LJ, Wu Y, et al. The Integrated Genomic and Epigenomic Landscape of Brainstem Glioma. Nat Commun (2020) 11:3077. doi: 10.1038/s41467-020-16682-y

5. Overview of the SEER Program. (2020). Available at: https://seer.cancer.gov/ about/overview.html (Accessed Jan, 2020).

6. Surveillance, Epidemiology, and End Results (SEER) Program. Seer ${ }^{\star}$ Stat Database: Incidence - SEER Research Data, 18 Registries, Nov 2019

\section{DATA AVAILABILITY STATEMENT}

The original contributions presented in the study are included in the article/supplementary material. Further inquiries can be directed to the corresponding authors.

\section{AUTHOR CONTRIBUTIONS}

YW and GZ: Conceptualization, methodology, and reviewing and editing. YW: Data curation, software, and validation. HL, $\mathrm{XQ}, \mathrm{LZ}$, and YW: Original draft preparation, and reviewing and editing. All authors contributed to the article and approved the submitted version.

Sub (2000-2017) - Linked to County Attributes - Time Dependent (1990-2017) Income/Rurality, 1969-2018 Counties. National Cancer Institute, DCCPS, Surveillance Research Program. Available at: www. seer.cancer.gov. released April 2020, based on the November 2019 submission.

7. Surveillance, Epidemiology, and End Results (SEER) Program. Seer ${ }^{\star}$ Stat Database: Incidence - SEER 18 Regs Custom Data (With Additional Treatment Fields), Nov 2018 Sub (1975-2016 Varying) - Linked to County Attributes - Total U.S., 1969-2017 Counties, National Cancer Institute, Dccps. Surveillance Research Program. Available at: www.seer.cancer.gov. released April 2019, based on the November 2018 submission.

8. Dellaretti M, Reyns N, Touzet G, Dubois F, Gusmão S, Pereira JL, et al. Diffuse Brainstem Glioma: Prognostic Factors. J Neurosurg (2012) 117:810-4. doi: 10.3171/2012.7.JNS111992

9. Hargrave D, Bartels U, Bouffet E. Diffuse Brainstem Glioma in Children: Critical Review of Clinical Trials. Lancet Oncol (2006) 7:241-8. doi: 10.1016/ S1470-2045(06)70615-5 
10. Laigle-Donadey F, Doz F, Delattre JY. Brainstem Gliomas in Children and Adults. Curr Opin Oncol (2008) 20:662-7. doi: 10.1097/CCO. ob013e32831186e 0

11. Maxwell R, Luksik AS, Garzon-Muvdi T, Yang W, Huang J, Bettegowda C, et al. Population-Based Study Determining Predictors of Cancer-Specific Mortality and Survival in Pediatric High-Grade Brainstem Glioma. World Neurosurg (2018) 119:e1006-15. doi: 10.1016/j.wneu.2018.08.044

12. Doyle J, Khalafallah AM, Yang W, Sun Y, Bettegowda C, Mukherjee D. Association Between Extent of Resection on Survival in Adult Brainstem High-Grade Glioma Patients. J Neuro Oncol (2019) 145:479-86. doi: 10.1007/ s11060-019-03313-w

13. Liu Z, Feng S, Li J, Cao H, Huang J, Fan F, et al. The Epidemiological Characteristics and Prognostic Factors of Low-Grade Brainstem Glioma: A Real-World Study of Pediatric and Adult Patients. Front Oncol (2020) 10:391. doi: 10.3389/fonc.2020.00391

14. Ostrom QT, Cioffi G, Gittleman H, Patil N, Waite K, Kruchko C, et al. Cbtrus Statistical Report: Primary Brain and Other Central Nervous System Tumors Diagnosed in the United States in 2012-2016. Neuro-Oncology (2019) 21:v1v100. doi: $10.1093 /$ neuonc/noz150

15. Smith MA, Freidlin B, Ries LA, Simon R. Trends in Reported Incidence of Primary Malignant Brain Tumors in Children in the United States. J Natl Cancer Inst (1998) 90:1269-77. doi: 10.1093/jnci/90.17.1269

16. Ostrom QT, Cote DJ, Ascha M, Kruchko C, Barnholtz-Sloan JS. Adult Glioma Incidence and Survival by Race or Ethnicity in the United States From 2000 to 2014. JAMA Oncol (2018) 4:1254-62. doi: 10.1001/ jamaoncol.2018.1789
17. Donaldson SS, Laningham F, Fisher PG. Advances Toward an Understanding of Brainstem Gliomas. J Clin Oncol Off J Am Soc Clin Oncol (2006) 24:126672. doi: 10.1200/JCO.2005.04.6599

18. Deland K, Starr BF, Mercer JS, Byemerwa J, Crabtree DM, Williams NT, et al. Tumor Genotype Dictates Radiosensitization After Atm Deletion in Primary Brainstem Glioma Models. J Clin Invest (2021) 131(1):e142158. doi: 10.1172/ JCI142158

19. Hu X, Fang Y, Hui X, Jv Y, You C. Radiotherapy for Diffuse Brainstem Glioma in Children and Young Adults. Cochrane Database Syst Rev (2016) 6: Cd010439. doi: 10.1002/14651858.CD010439.pub2

20. Osorio DS, Patel N, Ji L, Sposto R, Stanek J, Gardner SL, et al. Pre-Irradiation Intensive Induction and Marrow-Ablative Consolidation Chemotherapy in Young Children With Newly Diagnosed High-Grade Brainstem Gliomas: Report of the "Head-Start" I and II Clinical Trials. J Neuro Oncol (2018) 140:717-25. doi: 10.1007/s11060-018-03003-Z

Conflict of Interest: The authors declare that the research was conducted in the absence of any commercial or financial relationships that could be construed as a potential conflict of interest.

Copyright (c) $2021 \mathrm{Liu}, \mathrm{Qin}$, Zhao, Zhao and Wang. This is an open-access article distributed under the terms of the Creative Commons Attribution License (CC BY).

The use, distribution or reproduction in other forums is permitted, provided the original author(s) and the copyright owner(s) are credited and that the original publication in this journal is cited, in accordance with accepted academic practice. No use, distribution or reproduction is permitted which does not comply with these terms. 